\title{
THE CONSTITUENT EQUATIONS OF PIEZOELECTRIC CANTILEVERED THREE-LAYER ACTUATORS WITH VARIOUS EXTERNAL LOADS AND GEOMETRY
}

\author{
Grzegorz MieczKowski \\ Bialystok University of Technology, Bialystok, Poland \\ e-mail: g.mieczkowski@pb.edu.pl
}

\begin{abstract}
This paper presents test results for deformation conditions of three-layer, piezoelectric cantilever converters subjected to various electrical and mechanical boundary conditions. A general solution has been developed based on implementation of piezoelectric triple segments $(P T S)$ to the beam. A working mechanism and conditions for strain of the PTS segment have been determined. Basing on the general solution, for the cantilever actuator subjected to an external force (of single and dual PTS segments) and a uniform load (of single PTS segment), particular solutions have also been developed. Moreover, dimensionless frequencies of the oscillating motion for the analyzed converters have been determined. In the next step, the influence of such factors as length, quantity and position of PTS segments, their relative stiffness and unit weight on values of the obtained frequencies of vibration have been defined. The resulting analytical solutions have been compared with the developed FEM solution.
\end{abstract}

Keywords: piezoelectric bender, constitutive equations, deflection, analytical solutions

\section{Introduction}

Piezoelectric transducers have been used over the years in many devices. These are exploited as sensors (Ştefănescu, 2011), actuators (Tzou, 1999), energy harvesters (Liu et al., 2014) or dynamic eliminators (Przybyłowicz, 1999). Their working principle is based on the conversion of electric energy to mechanical or other way around (Bush-Vishniac, 1999). The relation between strain and electric field is defined by constitutive equations ((Curie and Curie, 1880; Berlincourt et al., 1964).

A significant aspect influencing the functionality and durability of converters is selection of a proper piezoelectric material. The properties of typical piezoelectric materials are presented in papers by Kawai (1969), Rajabi et al. (2015).

Another important factor is a static characteristic of the converter. When designing piezoelectric converters for specific application, it is necessary to define and solve adequate simultaneous equations. These equations bound together geometrical properties, material properties and physical parameters such as force, deflection and electric field. Solving such simultaneous equations is very difficult. Materials and geometrical inhomogeneity of the converter global structure and anisotropy of piezoelectric materials forces the use of some reductions. Smits et al. (1991), by using energetic methods, formed and solved constitutive equations for a converter made of two layers of even length (piezoelectric bimorph). In the paper by Wang and Cross (1999) there is an issue of a three-layer converter extended and solved, whereas in (Xiang and Shi, 2008) - a multi-layer one. The static characteristics of two-layer converters with different length of layers are shown in (Park and Moon, 2005; Raeisifard et al., 2014; Mieczkowski, 2016).

Piezoelectric converters as well as other elastic bodies having mass are prone to vibrations. Therefore, it is necessary to define the nature of their oscillating motion. Many authors have 
dealt with this issue. Chen et al.(1998), Askari Farsangi et al. (2013) studied free vibrations of piezoelectric laminated plates presented, whereas Clare et al. (1991)analysed a simply supported beam with piezoelectric patches. Also, analyses of dynamic characteristics of transducers where forced vibration occurred are shown in papers by Rouzegar and Abad (2015), Bleustein and Tiersten (1968), Djojodihardjo et al. (2015). Dynamical aspects of converters with the piezoelectric patches, including control strategy, were considered by Tylikowski (1993), Pietrzakowski (2000, 2001), Buchacz and Płaczek (2009).

It is a very rare case that in literature one can find results of tests describing the influence of geometrical and material characteristics, number and location of piezoelectric patches on static deflection and free vibration.

In such cases, in order to determine electromechanical behaviour of the converter, usually the FEM-based analyses are carried out, see (Rahmoune and Osmont, 2010; Mieszczak et al., 2006). However, carrying out this type of analyses is very work-consuming and the solution may be subjected to high error.

Therefore, the main purpose of the present paper is to develop a simple analytical method for determining deflection in function of mechanical and electric loads. By design, the piezoelectric converters have individual components (layers) of different length and the piezoelectric layer can be divided, which is an extension to the researches shown in papers by Smits et al. (1991), Wang and Cross (1999), Xiang and Shi (2008), where lengths of the beam element and piezoelectric layers were equal, see Fig. 1.

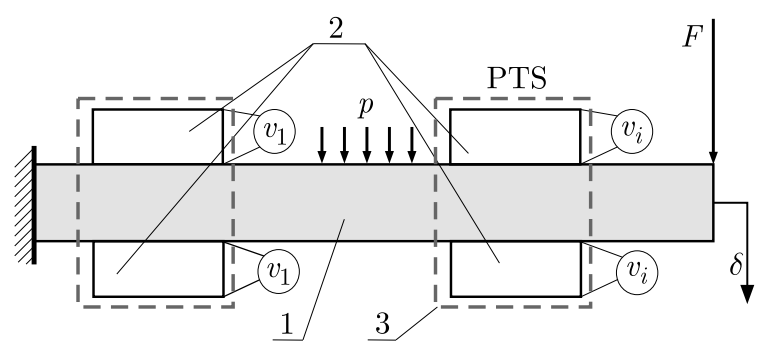

Fig. 1. Three-layer piezoelectric converter, 1 - beam, 2 - piezoelectric elements, 3 - piezoelectric triple segment PTS

The proposed method involves the implementation of modules to a homogeneous beam, further referred to as the piezoelectric triple segment (PTS). This allows including a local change in stiffness and strain caused by the transverse piezoelectric effect within the analytical description of the beam deflection.

In view of material and geometric discontinuity in the analyzed transducers, natural frequencies of oscillating motion turn out to be different than those in the homogeneous beam. Therefore, the next aim of the paper is analysis of dynamical behavior of such converters. In the present work, examination similar to that carried out by Clare et al. (1991) is conducted. It is extended by analysis of the influence of the number, length and location of piezoelectric segments and their material properties on the natural frequency values.

In order to verify the correctness of the solutions, it is required to compare the obtained results with experimental data or results obtained using other methods. Therefore, for converters with the diversified material and geometric structure, FEM simulations have been made and compared with the obtained analytical results. 


\section{Analytical results}

\subsection{Basic assumptions}

The converters analysed in this work are treated as a homogeneous (one-layer) beam with locally implemented piezoelectric triple segments PTS (Fig. 1). The PTS is made up of three components - two piezoelectric and one non-piezoelectric element. The non-piezoelectric layer thickness is the same as the beam thickness. The beam and the PTS both have the same width. In order to simplify the mathematical model, the following assumptions are made:

- bending of the element takes place according to Euler's hypothesis, and radii of curvatures of the deflected components are identical,

- in the connection plane between components there is no intermediate layer and no sliding occurs,

- in the piezoelectric layer, the transverse piezoelectric effect 1-3 takes place causing clear bending.

\subsection{General solution to the piezoelectric converter with implemented PTS segment}

The task is to consider a section of the piezoelectric converter (Fig. 2) subjected to mechanical bending moment $M(x)$ and electric moment $M_{e}$ (following the occurrence of the piezoelectric effect).

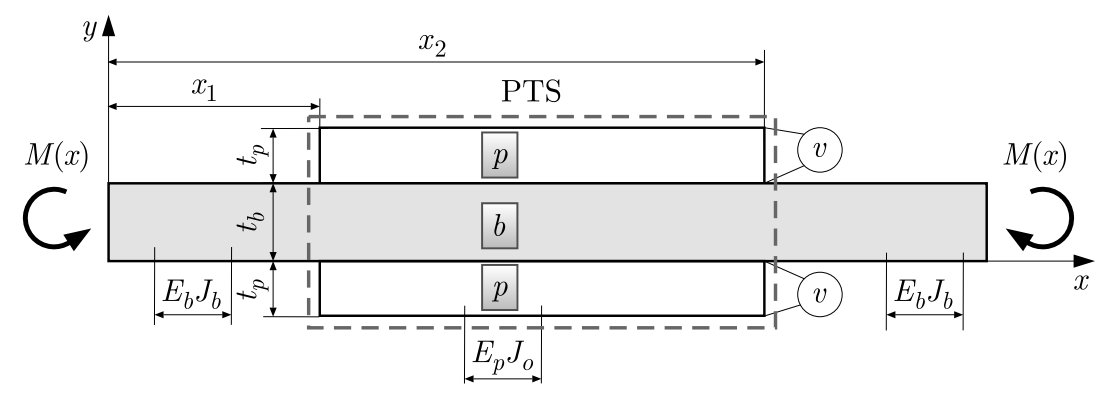

Fig. 2. Section of the piezoelectric converter

In the analysed element subjected to bending, it is possible to determine three characteristic ranges related to a change in load and stiffness. Within the $x_{1}<x<x_{2}$ range, there is a piezoelectric triple segment PTS (generating $M_{e}$ ) with flexural stiffness $E_{p} J_{o}$. The other two ranges are a homogeneous beam with stiffness $E_{b} J_{b}$. Since there are several characteristic ranges on the beam, it is convenient to make use of Heaviside's function. Thus, including the PTS presence in the beam, the deflection line can be described using the following dependence

$$
\frac{\partial^{2} y}{\partial x^{2}}=\frac{M(x)}{E_{b} J_{b}}+M_{e} \gamma\left(H\left[x-x_{1}\right]-H\left[x-x_{2}\right]\right)
$$

where: $H\left[x-x_{i}\right]-$ Heaviside's function, $E_{p}, E_{b}$ - Young's moduli of the piezoelectric and nonpiezoelectric element, $J_{b}, J_{o}$ - moments of inertia (described in Section 2.3), $\gamma$ - factor including the change in stiffness with applied formal notation of Heaviside's function

$$
\gamma=\frac{E_{b} J_{b}\left[M_{e}+M(x)\right]-E_{p} J_{o} M(x)}{E_{b} J_{b} E_{p} J_{o} M_{e}}
$$

As determining the mechanical moment $\mathrm{M}(\mathrm{x})$ in general does not pose any problems, determining the electrical load $M_{e}$ generated by the PTS is very burdensome and requires solving the two-dimension problem of the PTS bending. 


\subsection{Piezoelectric triple segment $P T S$}

The task is to consider the segment PTS (Fig. 3) with constant width $b$ consisting of non-piezoelectric (2) and piezoelectric layers (1) and (3).

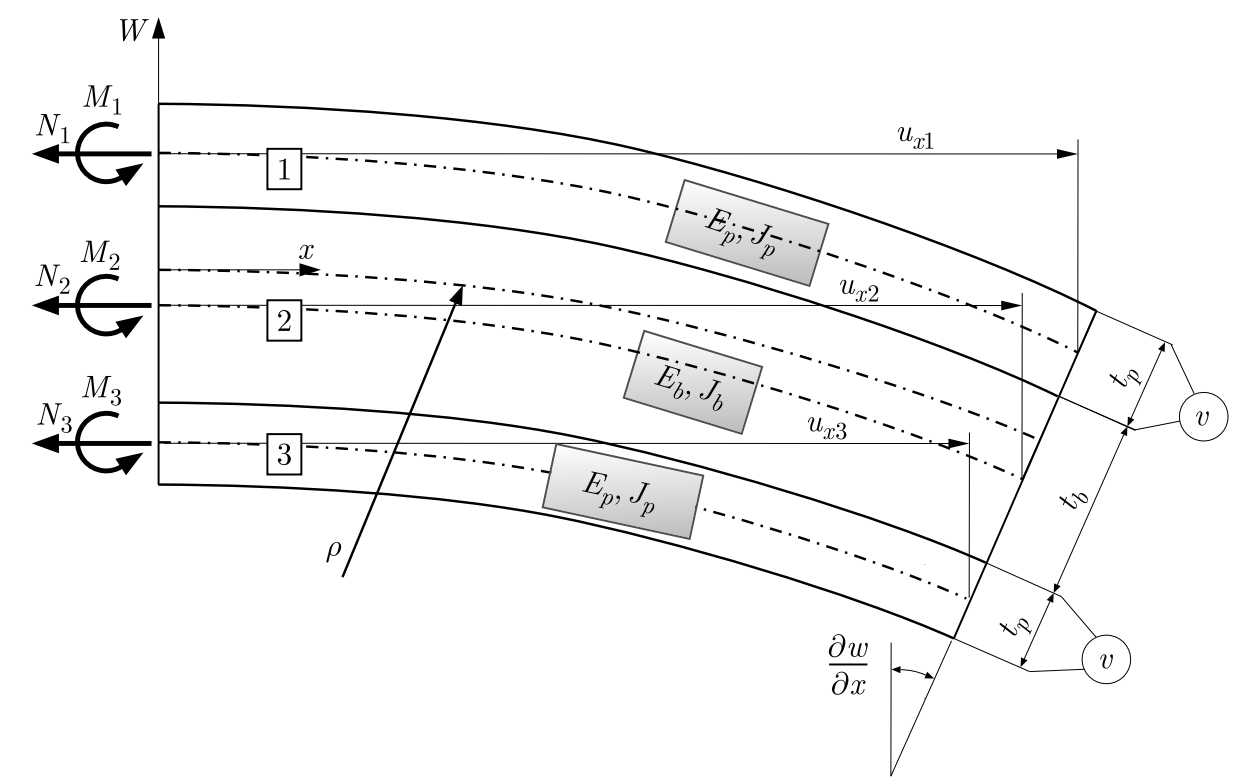

Fig. 3. Distribution of forces and conditions for strain of the piezoelectric triple segment PTS

The structure is not subjected to any mechanical load. The longitudinal forces $N_{i}$ and bending moments $M_{i}$ occurring in individual layers are a result of the applied voltage $v$. Basing on the equilibrium equation of forces. the following can be rritten

$$
N_{1}+N_{2}+N_{3}=0
$$

The sum of moments in relation to the upper interface must be zero, therefore

$$
M_{1}+M_{2}+M_{3}-\frac{N_{2} t_{b}}{2}-N_{3}\left(t_{b}+\frac{t_{p}}{2}\right)+\frac{N_{1} t_{p}}{2}=0
$$

According to the adopted Euler hypothesis, bending moments can be described as follows

$$
M_{1}=\frac{E_{p}}{J_{p}} \rho \quad M_{2}=\frac{E_{b}}{J_{b}} \rho \quad M_{3}=\frac{E_{p}}{J_{p}} \rho
$$

Substituting dependences (2.4) to (2.3) and making simple transformations results in the following

$$
\frac{1}{\rho}=\frac{\left(N_{2}+2 N_{3}\right) t_{b}+\left(N_{3}-N_{1}\right) t_{p}}{2 E_{b} J_{b}+4 E_{p} J_{p}}
$$

Including the relation between the radius of curvature $\rho$ and deflection $w(x)$

$$
\frac{1}{\rho}=\frac{\partial^{2} w}{\partial x^{2}}
$$

the differential equation for the converter bending can be found as follows

$$
\frac{\partial^{2} w}{\partial x^{2}}=\frac{\left(N_{2}+2 N_{3}\right) t_{b}+\left(N_{3}-N_{1}\right) t_{p}}{2 E_{b} J_{b}+4 E_{p} J_{p}}
$$


The constitutive equations for all converter layers, including the piezoelectric effect in layer 1 and 3 give the following

$$
\frac{\partial u_{x 1}}{\partial x}=\frac{N_{1}}{E_{p} A_{p}}-d_{31}\left(\frac{-v}{t_{p}}\right) \quad \frac{\partial u_{x 2}}{\partial x}=\frac{N_{2}}{E_{b} A_{b}} \quad \frac{\partial u_{x 3}}{\partial x}=\frac{N_{3}}{E_{p} A_{p}}+d_{31}\left(\frac{-v}{t_{p}}\right)
$$

where $A_{b}=t_{b} b, A_{p}=t_{p} b$ are layers cross sectional areas, $d_{31}$ - piezoelectric constant.

Following the relocation continuity condition (Fig. 3), it is found that

$$
u_{x 1}-u_{x 2}-\frac{\partial w}{\partial x}\left(\frac{t_{p}}{2}+\frac{t_{b}}{2}\right)=0 \quad u_{x 1}-u_{x 3}-\frac{\partial w}{\partial x}\left(\frac{t_{p}}{2}+\frac{t_{b}}{2}+t_{b}\right)=0
$$

Solving differential equations (2.7) and (2.8) with the following boundary conditions

$$
\begin{array}{lll}
\frac{\partial w}{\partial x}(0)=0 & w(0)=0 & \\
u_{x 1}(0)=0 & u_{x 2}(0)=0 & u_{x 3}(0)=0
\end{array}
$$

and applying dependence (2.9) and (2.2), the longitudinal force $N_{i}$ can be determined

$$
N_{1}=\frac{-b E_{p} v d_{31}\left(E_{b} t_{b}^{3}+2 E_{p} t_{p}^{3}\right)}{\alpha} \quad N_{2}=0 \quad N_{3}=\frac{b E_{p} v d_{31}\left(E_{b} t_{b}^{3}+2 E_{p} t_{p}^{3}\right)}{\alpha}
$$

where: $\alpha=E_{b} t_{b}^{3}-2 E_{p} t_{p}\left(3 t_{b}^{2}+6 t_{b} t_{p}+2 t_{p}^{2}\right)$.

The differential equation for bending PTS in the $M_{e}$ moment function can be written as follows

$$
\frac{\partial^{2} w}{\partial x^{2}}=\frac{-2 M_{e}}{E_{p} J_{o}}
$$

On the basis of comparing equations (2.7) and (2.12), it is possible to determine the bending moment $M_{e}$ which results from the piezoelectric effect

$$
M_{e}=\frac{E_{p} J_{o}\left[-\left(N_{2}+2 N_{3}\right) t_{b}+\left(N_{1}-N_{3}\right) t_{p}\right]}{4 E_{b} J_{b}+8 E_{p} J_{p}}
$$

where moments of inertia for the individual layers are, respectively

$$
J_{b}=\frac{b t_{b}^{3}}{12} \quad J_{p}=\frac{b t_{p}^{3}}{12}
$$

The averaging value of the moment of inertia $J_{o}$ can be calculated using the method of transformation of the cross sectional area (Fertis, 1996). Three materials of different stiffness moduli and the same width $b$ (Fig. 4a) are replaced with one material of the section composed of three parts of different widths (Fig. 4b).

The sought moment of inertia, calculated in relation to the neutral layer, is

$$
J_{o}=\frac{b \beta}{12 E_{p}}
$$

where: $\beta=E_{b} t_{b}^{3}+2 E_{p} t_{p}\left(3 t_{b}^{2}+6 t_{b} t_{p}+4 t_{p}^{2}\right)$.

Substituting formula (2.13) with (2.11), (2.14) and (2.15) results in the electric bending moment value in function of the applied voltage $v$

$$
M_{e}=-\frac{b E_{p} v \beta d_{31}\left(t_{b}+t_{p}\right)}{2 \alpha}
$$


(a)

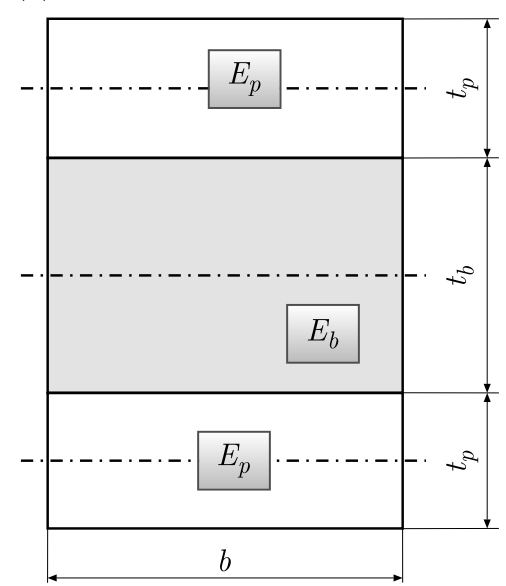

(b)

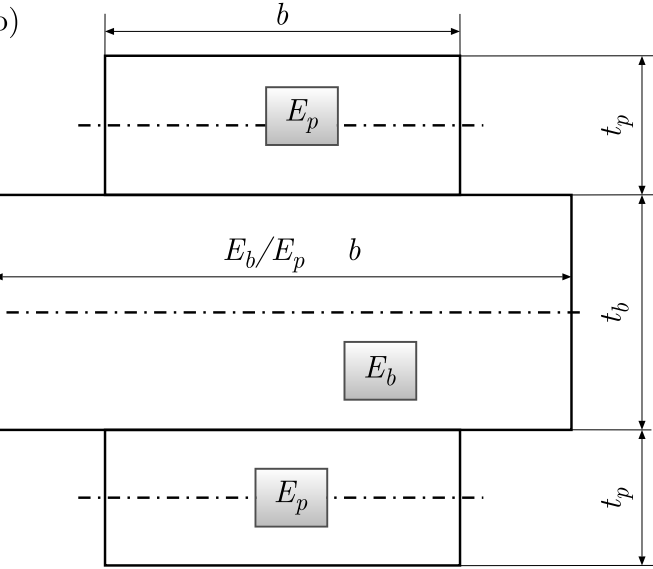

Fig. 4. Original (a) and transformed (b) section in the piezoelectric triple segment PTS

\subsection{Particular solutions}

This part of the work is concerned with the application of the proposed method based on implementing PTS segments into the single-layer beam to determine analytical dependences describing bending of the converters of fixed geometry and known boundary conditions. Solutions for the converters of different external loads and the PTS number shall be presented.

\subsubsection{Cantilever converter subjected to concentrated force $F$ of a single PTS segment}

In the converter, as shown in Fig. 5, the left end is fixed and the right end can move freely. The load results from the external force $F$ and the electric moment $M_{e}$ is generated by the applied voltage $v$. Based on the conditions for equilibrium of forces and moments, the reactions in the mounting are established: $R_{y}=F, R_{x}=0, M_{F}=F L$.

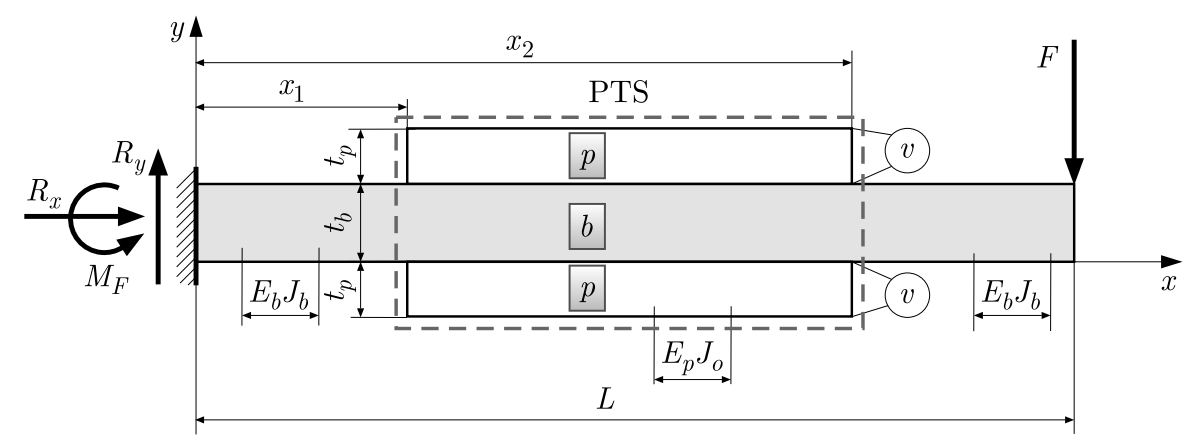

Fig. 5. Cantilever converter of the single PTS segment

The mechanical moment $M(x)$ takes the following form

$$
M(x)=-M_{F}+R_{y} x=-F L+F x
$$

Substituting expressions (2.16) and (2.17) to the general solution described with formula (2.1), upon double integration gives a dependence describing the function of bending of the analysed converter

$$
y(x)=A_{1} v+B_{1} F
$$

where 


$$
\begin{aligned}
A_{1} & =\frac{-3 d_{31} E_{p}\left(t_{b}+t_{p}\right)}{\alpha}\left(H\left[x-x_{1}\right]\left(x-x_{1}\right)^{2}-H\left[x-x_{2}\right]\left(x-x_{2}\right)^{2}\right) \\
B_{1} & =\frac{2}{b \beta E_{b} t_{b}^{3}}\left\{( \beta - E _ { b } t _ { b } ^ { 3 } ) \left(H\left[x-x_{1}\right]\left(3 L-x-2 x_{1}\right)\left(x-x_{1}\right)^{2}\right.\right. \\
& \left.\left.-H\left[x-x_{2}\right]\left(3 L-x-2 x_{2}\right)\left(x-x_{2}\right)^{2}\right)-\beta x^{2}(3 L-x)\right\}
\end{aligned}
$$

The integration constants are determined on the basis of the following boundary conditions

$$
\frac{\partial y}{\partial x}(0)=0 \quad y(0)=0
$$

\subsubsection{Cantilever converter subjected to concentrated force $F$ of two PTS segments}

For the converter shown in Fig. 6 the conditions of mounting and mechanical load are identical as in the case described in Section 2.4.1. The electrical load is generated by two PTS segments powered by voltage $v_{1}$ and $v_{2}$.

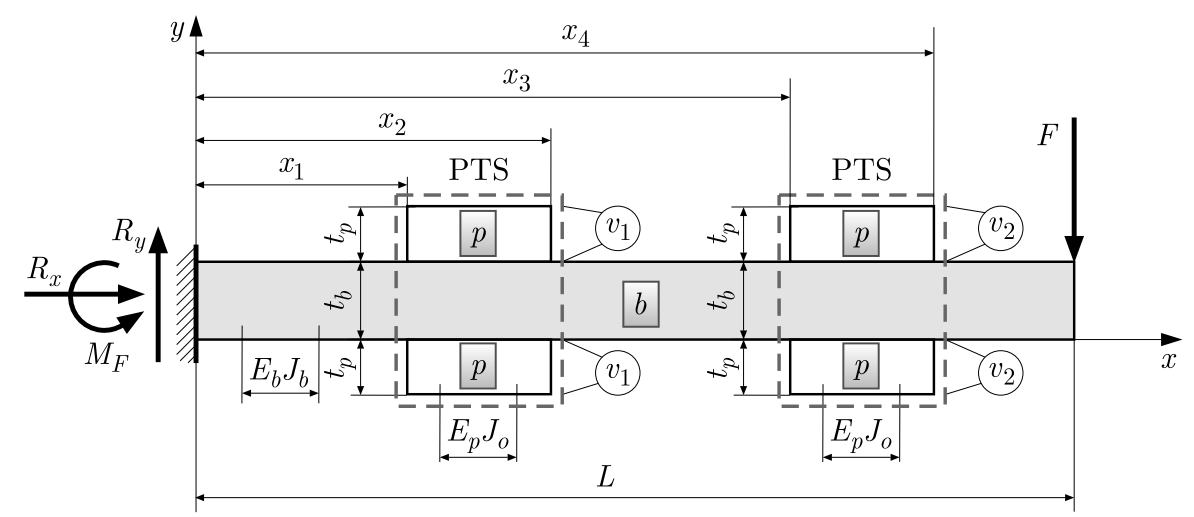

Fig. 6. Cantilever converter of two PTS segments

The differential equation for deflection is as follows

$$
\frac{\partial^{2} y}{\partial x^{2}}=\frac{M(x)}{E_{b} J_{b}}+M e_{1} \gamma\left(H\left[x-x_{1}\right]-H\left[x-x_{2}\right]\right)+M e_{2} \gamma\left(H\left[x-x_{3}\right]-H\left[x-x_{4}\right]\right)
$$

In the formula above, the mechanical moment $M(x)$ is described by equation $(2.17)$, while the electrical moments are

$$
M_{e}=-\frac{b E_{p} v_{i} \beta d_{31}\left(t_{b}+t_{p}\right)}{2 \alpha} \quad i=1,2
$$

Solving differential equation (2.20) and assuming boundary conditions (2.19) gives the function describing bending of the analysed converter

$$
y(x)=A_{1} v_{1}+A_{2} v_{2}+B_{2} F
$$

where

$$
\begin{aligned}
A_{2} & =\frac{-3 d_{31} E_{p}\left(t_{b}+t_{p}\right)}{\alpha}\left(H\left[x-x_{3}\right]\left(x-x_{3}\right)^{2}-H\left[x-x_{4}\right]\left(x-x_{4}\right)^{2}\right) \\
B_{2} & =\frac{2}{b \beta E_{b} t_{b}^{3}}\left\{( \beta - E _ { b } t _ { b } ^ { 3 } ) \left(H\left[x-x_{1}\right]\left(3 L-x-2 x_{1}\right)\left(x-x_{1}\right)^{2}\right.\right. \\
& -H\left[x-x_{2}\right]\left(3 L-x-2 x_{2}\right)\left(x-x_{2}\right)^{2}+H\left[x-x_{3}\right]\left(3 L-x-2 x_{3}\right)\left(x-x_{3}\right)^{2} \\
& \left.\left.-H\left[x-x_{4}\right]\left(3 L-x-2 x_{4}\right)\left(x-x_{4}\right)^{2}\right)-\beta x^{2}(3 L-x)\right\}
\end{aligned}
$$




\subsubsection{Cantilever converter subjected to uniform external load $p$ of the single PTS segment}

In the actuator shown in Fig. 7, the conditions of mounting are identical as those described in Sections 2.4.1 and 2.4.2. The converter is acted on by a uniform load $p$. The electrical moment is generated by a single PTS located at the left end.

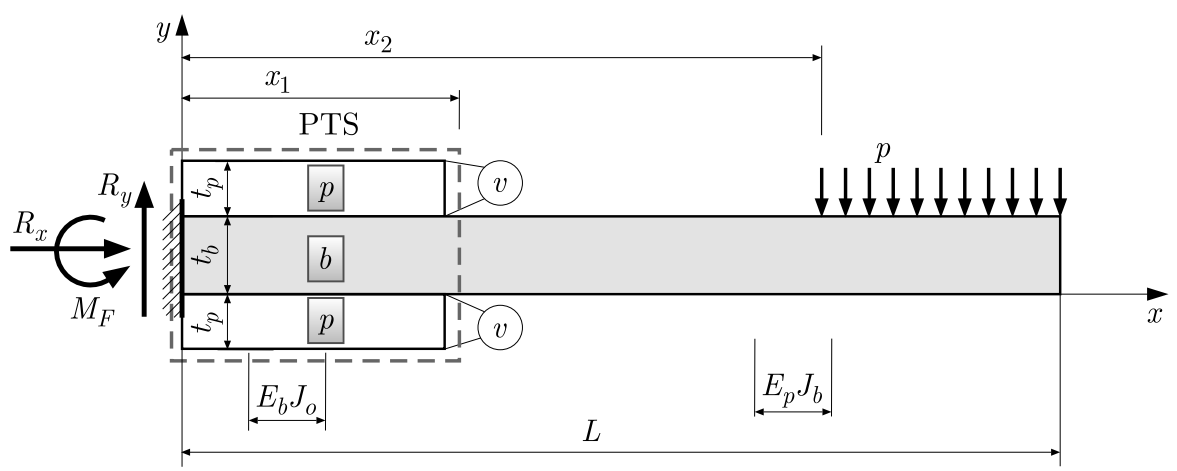

Fig. 7. Cantilever converter of a single PTS segment

Based on the conditions for equilibrium of forces and moments, the reactions in the mounting are determined: $R_{y}=p\left(L-x_{2}\right), M_{F}=0.5 p\left(L-x_{2}\right)\left(L+x_{2}\right)$. The mechanical moment $M(x)$ takes the following form

$$
M(x)=R_{y} x-M_{F}-\frac{p\left(x-x_{2}\right)^{2}}{2} H\left[x-x_{2}\right]
$$

Solving differential equation (2.1), assuming mechanical (2.23) and electric (2.16) moments gives the equation describing deflection of the analysed converter

$$
y(x)=A_{3} v+B_{3} p
$$

where

$$
\begin{aligned}
A_{3} & =\frac{-3 d_{31} E_{p}\left(t_{b}+t_{p}\right)}{\alpha}\left(H[x] x^{2}-H\left[x-x_{1}\right]\left(x-x_{1}\right)^{2}\right) \\
B_{3} & =\frac{1}{2 b \beta E_{b} t_{b}^{3}}\left\{\left(\beta-E_{b} t_{b}^{3}\right)\right. \\
& \cdot\left(H\left[x-x_{1}\right]\left(H\left[x-x_{2}\right]\left(x-x_{2}\right)^{4}+2\left(x-x_{1}\right)^{2}\left(L-x_{2}\right)\left(3 L-2 x+3 x_{2}\right)\right)\right. \\
& \left.-H[x]\left(H\left[x-x_{2}\right]\left(x-x_{2}\right)^{4}+2 x^{2}\left(L-x_{2}\right)\left(3 L-2 x+3 x_{2}\right)\right)\right) \\
& \left.+\beta\left(H\left[x-x_{2}\right]\left(x-x_{2}\right)^{4}+2 x^{2}\left(L-x_{2}\right)\left(3 L-2 x+3 x_{2}\right)\right)\right\}
\end{aligned}
$$

Integration constants are determined on the basis of the following boundary conditions

$$
\frac{\partial y}{\partial x}(0)=0 \quad y(0)=0
$$

\subsection{Dynamical behavior of piezoelectric converters}

As mentioned before, piezoelectric transducers similarly to other elastic bodies having mass, are prone to vibrations. In this respect, it is advised to include its oscillating nature of motion in the process of designing and exploitation of piezoelectric structures. Generally, vibrations can be divided into two groups - free and forced. Free vibrations occur when external forces do not influence the body and the system vibrates due to action of inherent forces. In that case, the 
system is going to vibrate with one or more natural frequencies. In vibrating systems natural damping occurs caused by forces of the internal friction. Damping is usually slight, thus does not affect the natural frequencies.

In the case when vibrations are caused by external forces, there appear the so called forced vibrations, and the system is going to vibrate with the excitation frequency.

The phenomenon of resonance is greatly dangerous for a structure. It occurs when the excitation frequency coincides with one of the natural frequencies which causes perilously high oscillations that may lead to damaging of the structure. Therefore, it is necessary to determine the natural frequencies and geometrical and material factors affecting their distribution.

Upon elementary theory of bending beams, equation (1) can be presented in the following way

$$
\frac{\partial^{2}}{\partial x^{2}}\left(E(x) J(x) \frac{\partial^{2} y}{\partial x^{2}}\right)+\rho(x) A(x) \frac{\partial^{2} y}{\partial t^{2}}=\frac{\partial^{2} M(x)}{\partial x^{2}}+M_{e}\left(\delta^{\prime}\left[x-x_{1}\right]-\delta^{\prime}\left[x-x_{2}\right]\right)
$$

where $\delta[x-x i], E(x), J(x), \rho(x), A(x)$ is the derivative of Dirac's function, Young's modulus, moment of inertia, density and cross sectional area of the converter. For free vibration, the exactly same differential equation can be written in the following way

$$
\frac{\partial^{2}}{\partial x^{2}}\left(E(x) J(x) \frac{\partial^{2} y}{\partial x^{2}}\right)+\rho(x) A(x) \frac{\partial^{2} y}{\partial t^{2}}=0
$$

The solution to equation (2.27) using the method of separation of variables can be written in the following way

$$
y(x, t)=W(x) T(t)
$$

where $W(x)$ is a function of space, and $T(t)$ depends only on time. Substituting (2.28) with (2.27) and performing simple mathematical modifications, the commonly known differential equation describing the beam boundary problem is obtained

$$
\frac{\partial^{2}}{\partial x^{2}}\left(E(x) J(x) \frac{\partial^{2} W}{\partial x^{2}}\right)-\rho(x) A(x) \omega^{2}=0
$$

where $\omega$ is the natural frequency of vibration.

Let us consider the piezoelectric transducer shown in Fig. 8.

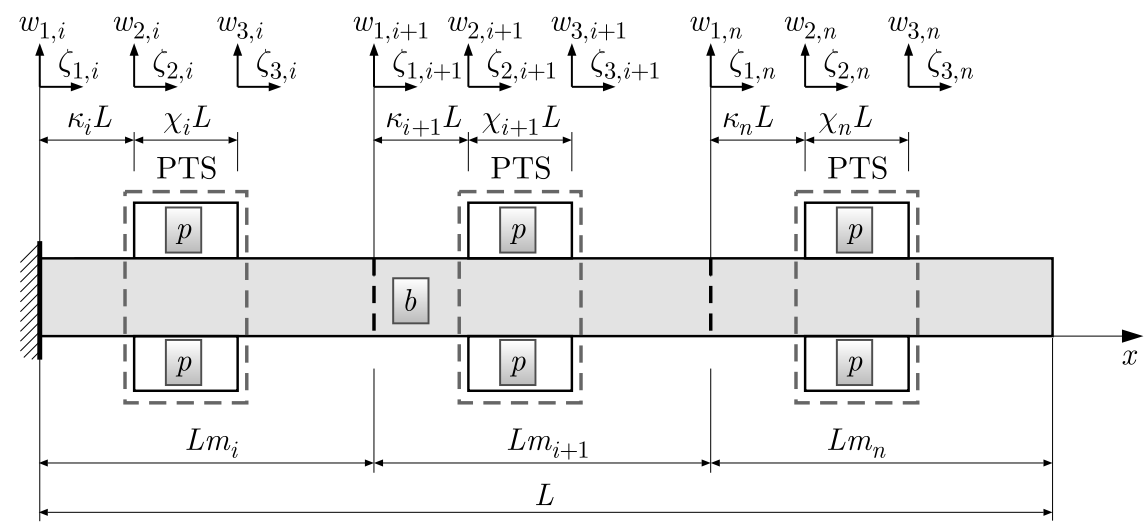

Fig. 8. Cantilever converter of $n$ PTS segment

There can be $n$ fragments distributed on the transducer whose total length is equal to $\sum_{i=1}^{n} L m_{i}=L$. Each and every fragment consists of three elements - a PTS segment and 
two beam elements. Applying local frames of reference, using the dimensionless coordinates, differential equation (2.29) is equivalent to

$$
\begin{array}{ll}
\frac{\partial^{4} W_{1, i}\left(\zeta_{1, i}\right)}{\partial \zeta_{1, i}^{4}}-\psi^{4} W_{1, i}\left(\zeta_{1, i}\right)=0 & \text { for } \quad \zeta_{1, i} \in\left\langle 0, \kappa_{i}\right\rangle \\
\frac{\partial^{4} W_{2, i}\left(\zeta_{2, i}\right)}{\partial \zeta_{2, i}^{4}}-\Lambda^{4} \psi^{4} W_{2, i}\left(\zeta_{2, i}\right)=0 & \text { for } \quad \zeta_{2, i} \in\left\langle 0, \chi_{i}\right\rangle \\
\frac{\partial^{4} W_{3, i}\left(\zeta_{3, i}\right)}{\partial \zeta_{3, i}^{4}}-\psi^{4} W_{3, i}\left(\zeta_{3, i}\right)=0 & \text { for } \quad \zeta_{3, i} \in\left\langle 0, \frac{L m_{i}}{L}-\kappa_{i}-\chi_{i}\right\rangle
\end{array}
$$

where

$$
\begin{aligned}
& \psi^{4}=L^{4} \omega^{2} \frac{\rho_{b} A_{b}}{E_{b} J_{b}} \quad \Lambda^{4}=\frac{E_{b} J_{b}}{\rho_{b} A_{b}} \frac{\rho_{b} A_{b}+2 \rho_{p} A_{p}}{E_{p} J_{o}} \\
& \zeta_{1, i}=\frac{x}{L} \quad \zeta_{2, i}=\frac{x-\kappa_{i} L}{L} \quad \zeta_{3, i}=\frac{x-\kappa_{i} L-\chi_{i} L}{L} \\
& \zeta_{1, i+1}=\frac{x-L m_{i}}{L} \quad i=1, \ldots, n
\end{aligned}
$$

$\omega$ is the natural frequency, $\rho_{b}, \rho_{p}$ is density of beam and piezoelectric material, respectively.

Solutions to differential equations (2.30) can be obtained as (Mahmoud and Nassar, 2000)

$$
\begin{aligned}
& W_{1, i}\left(\zeta_{1, i}\right)=A_{1, i} \sin \left(\psi \zeta_{1, i}\right)+B_{1, i} \cos \left(\psi \zeta_{1, i}\right)+C_{1, i} \cosh \left(\psi \zeta_{1, i}\right)+D_{1, i} \sinh \left(\psi \zeta_{1, i}\right) \\
& W_{2, i}\left(\zeta_{2, i}\right)=A_{2, i} \sin \left(\Lambda \psi \zeta_{2, i}\right)+B_{2, i} \cos \left(\Lambda \psi \zeta_{2, i}\right)+C_{2, i} \cosh \left(\Lambda \psi \zeta_{2, i}\right)+D_{2, i} \sinh \left(\Lambda \psi \zeta_{2, i}\right) \\
& W_{3, i}\left(\zeta_{3, i}\right)=A_{3, i} \sin \left(\psi \zeta_{3, i}\right)+B_{3, i} \cos \left(\psi \zeta_{3, i}\right)+C_{3, i} \cosh \left(\psi \zeta_{3, i}\right)+D_{3, i} \sinh \left(\psi \zeta_{3, i}\right)
\end{aligned}
$$

where $A_{j, i}, B_{j, i}, C_{j, i}, D_{j, i}, j=1,2,3$ are constants.

The boundary conditions of mounting of the converter (left end is fix-mounted and the right one freely move) together with continuity conditions at the intermediate ends lead to a set $n \times 12$ linear homogeneous equations.

The continuity conditions adopt the following form

$$
\begin{aligned}
& \left.W_{1, i}\left(\zeta_{1, i}\right)\right|_{\zeta_{1, i}=\kappa_{i}}=\left.\left.W_{2, i}\left(\zeta_{2, i}\right)\right|_{\zeta_{2, i}=0} \quad \frac{\partial W_{1, i}\left(\zeta_{1, i}\right)}{\partial \zeta_{1, i}}\right|_{\zeta_{1, i}=\kappa_{i}}=\left.\frac{\partial W_{2, i}\left(\zeta_{2, i}\right)}{\partial \zeta_{1, i}}\right|_{\zeta_{2, i}=0} \\
& \left.\frac{\partial^{2} W_{1, i}\left(\zeta_{1, i}\right)}{\partial \zeta_{1, i}^{2}}\right|_{\zeta_{1, i}=\kappa_{i}}=\left.\eta \frac{\partial^{2} W_{2, i}\left(\zeta_{2, i}\right)}{\partial \zeta_{2, i}^{2}}\right|_{\zeta_{2, i}=0} \\
& \left.\frac{\partial^{3} W_{1, i}\left(\zeta_{1, i}\right)}{\partial \zeta_{1, i}^{3}}\right|_{\zeta_{1, i}=\kappa_{i}}=\left.\eta \frac{\partial^{3} W_{2, i}\left(\zeta_{2, i}\right)}{\partial \zeta_{2, i}^{3}}\right|_{\zeta_{2, i}=0} \\
& \left.W_{2, i}\left(\zeta_{2, i}\right)\right|_{\zeta_{2, i}=\chi_{i}}=\left.\left.W_{3, i}\left(\zeta_{3, i}\right)\right|_{\zeta_{3, i}=0} \quad \frac{\partial W_{2, i}\left(\zeta_{2, i}\right)}{\partial \zeta_{2, i}}\right|_{\zeta_{2, i}=\chi_{i}}=\left.\frac{\partial W_{3, i}\left(\zeta_{3, i}\right)}{\partial \zeta_{3, i}}\right|_{\zeta_{3, i}=0} \\
& \left.\eta \frac{\partial^{2} W_{2, i}\left(\zeta_{2, i}\right)}{\partial \zeta_{2, i}^{2}}\right|_{\zeta_{2, i}=\chi_{i}}=\left.\frac{\partial^{2} W_{3, i}\left(\zeta_{3, i}\right)}{\partial \zeta_{3, i}^{2}}\right|_{\zeta_{3, i}=0} \\
& \left.\eta \frac{\partial^{3} W_{2, i}\left(\zeta_{2, i}\right)}{\partial \zeta_{2, i}^{3}}\right|_{\zeta_{2, i}=\chi_{i}}=\left.\frac{\partial^{3} W_{3, i}\left(\zeta_{3, i}\right)}{\partial \zeta_{3, i}^{3}}\right|_{\zeta_{3, i}=0}
\end{aligned}
$$




$$
\begin{aligned}
& \left.W_{3, i}\left(\zeta_{3, i}\right)\right|_{\zeta_{3, i}=\phi_{i}}=\left.W_{1, i+1}\left(\zeta_{1, i+1}\right)\right|_{\zeta_{1, i+1}=0} \\
& \left.\frac{\partial W_{3, i}\left(\zeta_{3, i}\right)}{\partial \zeta_{3, i}}\right|_{\zeta_{3, i}=\phi_{i}}=\left.\frac{\partial W_{1, i+1}\left(\zeta_{1, i+1}\right)}{\partial \zeta_{1, i+1}}\right|_{\zeta_{1, i+1}=0} \\
& \left.\frac{\partial^{2} W_{3, i}\left(\zeta_{3, i}\right)}{\partial \zeta_{3, i}^{2}}\right|_{\zeta_{3, i}=\phi_{i}}=\left.\frac{\partial^{2} W_{1, i+1}\left(\zeta_{1, i+1}\right)}{\partial \zeta_{1, i+1}^{2}}\right|_{\zeta_{1, i+1}=0} \\
& \left.\frac{\partial^{3} W_{3, i}\left(\zeta_{3, i}\right)}{\partial \zeta_{3, i}^{3}}\right|_{\zeta_{3, i}=\phi_{i}}=\left.\frac{\partial^{3} W_{1, i+1}\left(\zeta_{1, i+1}\right)}{\partial \zeta_{1, i+1}^{3}}\right|_{\zeta_{1, i+1}=0}
\end{aligned}
$$

where

$$
\eta=\frac{E_{p} J_{o}}{E_{b} J_{b}} \quad \phi_{i}=\frac{L m_{i}}{L}-\kappa_{i}-\chi_{i}
$$

The boundary conditions can be written as follows:

- fixed

$$
W_{1,1}\left(\zeta_{1,1}\right)=\left.0\right|_{\zeta_{1,1}=0} \quad \frac{\partial W_{1,1}\left(\zeta_{1,1}\right)}{\partial \zeta_{1,1}}=\left.0\right|_{\zeta_{1,1}=0}
$$

- free

$$
E_{b} J_{b} \frac{\partial^{2} W_{3, n}\left(\zeta_{3, n}\right)}{\partial \zeta_{3, n}^{2}}=\left.0\right|_{\zeta_{3, n}=\frac{L m_{n}}{L}-\kappa_{n}-\chi_{n}} \quad E_{b} J_{b} \frac{\partial^{3} W_{3, n}\left(\zeta_{3, n}\right)}{\partial \zeta_{3, n}^{3}}=\left.0\right|_{\zeta_{3, n}=\frac{L m_{n}}{L}-\kappa_{n}-\chi_{n}}
$$

Using dependences (2.31)-(2.34), as mentioned before, $n \times 12$ linear homogenous equation can be achieved.

The values of dimensionless frequencies $\psi$ are determined from the characteristic equation representing the zero determinant of the matrix of boundary conditions $\mathbf{M}^{12 n \times 12 n}$

$$
\mathbf{M}^{12 n \times 12 n}=\left[\begin{array}{cccc}
\mathbf{M}_{i}^{12 \times 12} & \mathbf{B}^{12 \times 12} & \ldots & \mathbf{0}^{12 \times 12} \\
\mathbf{C}_{i}^{12 \times 12} & \mathbf{M}_{i+1}^{12 \times 12} & \ldots & \mathbf{0}^{12 \times 12} \\
\vdots & \vdots & \ddots & \mathbf{B}^{12 \times 12} \\
\mathbf{0}^{12 \times 12} & \mathbf{0}^{12 \times 12} & \mathbf{C}_{n-1}^{12 \times 12} & \mathbf{M}_{n}^{12 \times 12}
\end{array}\right]
$$

where $\mathbf{0}^{12 \times 12}$ is the zero matrix, $\mathbf{M}_{i}^{12 \times 12}=\left[\begin{array}{lll}\mathbf{M}_{1, i}^{12 \times 4} & \mathbf{M}_{2, i}^{12 \times 4} & \mathbf{M}_{2, i}^{12 \times 4}\end{array}\right]$, the remaining matrices are shown in Appendix. For the transducer with one segment, matrix (2.35) simplifies to the following form

$$
\mathbf{M}^{12 \times 12}=\left[\begin{array}{lll}
\mathbf{M}_{1,1}^{12 \times 4} & \mathbf{M}_{2,1}^{12 \times 4} & \mathbf{M}_{2,1}^{12 \times 4}
\end{array}\right]
$$

An analytical form of the characteristic equation $\left(\left|\mathbf{M}^{12 n x 12 n}\right|=0\right)$ in special cases where $\sum_{i=1}^{n} \chi_{i}=0$ (homogenous beam) and $\sum_{i=1}^{n} \chi_{i}=1$ (PTS segment all along) is described from subsequent equations

$$
1+\cos \psi \cosh \psi=0 \quad 1+\cos (\Lambda \psi) \cosh (\Lambda \psi)=0
$$

In other cases, in order to determine $\psi$, the roots of the characteristic equation can be obtained using numerical methods. In Figs. 9 and 10, there are dimensionless frequencies $\psi$ presented in function of length of the piezoelectric layer for different locations and amount of PTS segments. The results achieved for the transducer with one PTS segment $\left(L m_{1}=L\right)$ are shown in Fig. 9, whereas for the transducer with two PTS segments $\left(L m_{1}+L m_{2}=L, L m_{1}=L m_{2}\right)$ are shown in Fig. 10. Furthermore, three variants of piezoelectric segment locations in the transducer have been examined. Namely: 
- PTS segment located on the left end $\left(\kappa_{i}=0\right)$,

- transducer with PTS segment located in the middle $\left(\kappa_{i}+0.5 \chi_{i}=0.25\right)$,

- PTS segment located on the right end $\left(\kappa_{i}+\chi_{i}=1\right)$.

Moreover, in the transducer with two piezoelectric segments there are identical geometric and material features adopted for both PTS segments.

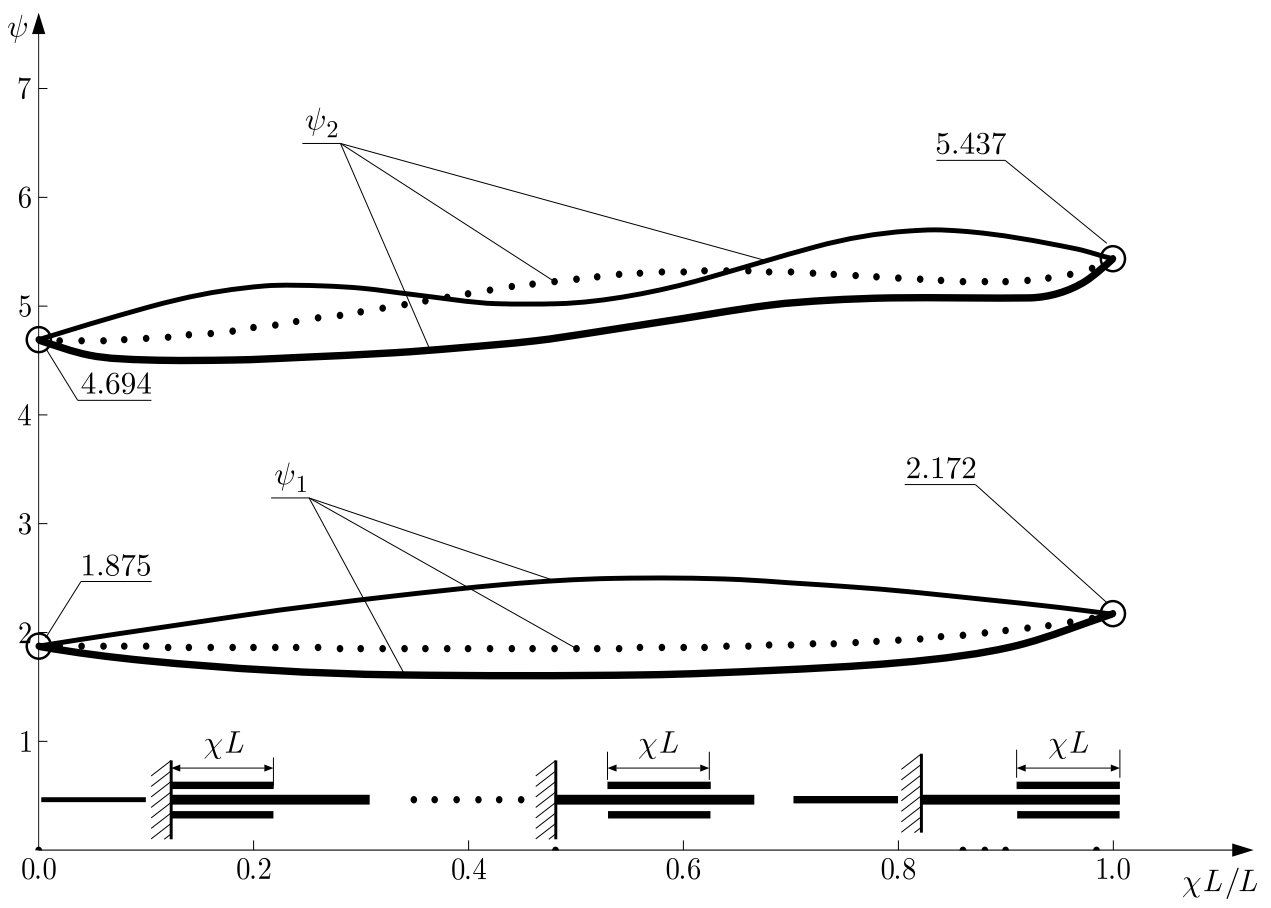

Fig. 9. The dimensionless frequencies $\psi$ for the converter eith a single PTS segment, $\psi_{1}$ - first frequency, $\psi_{2}$ - second frequency

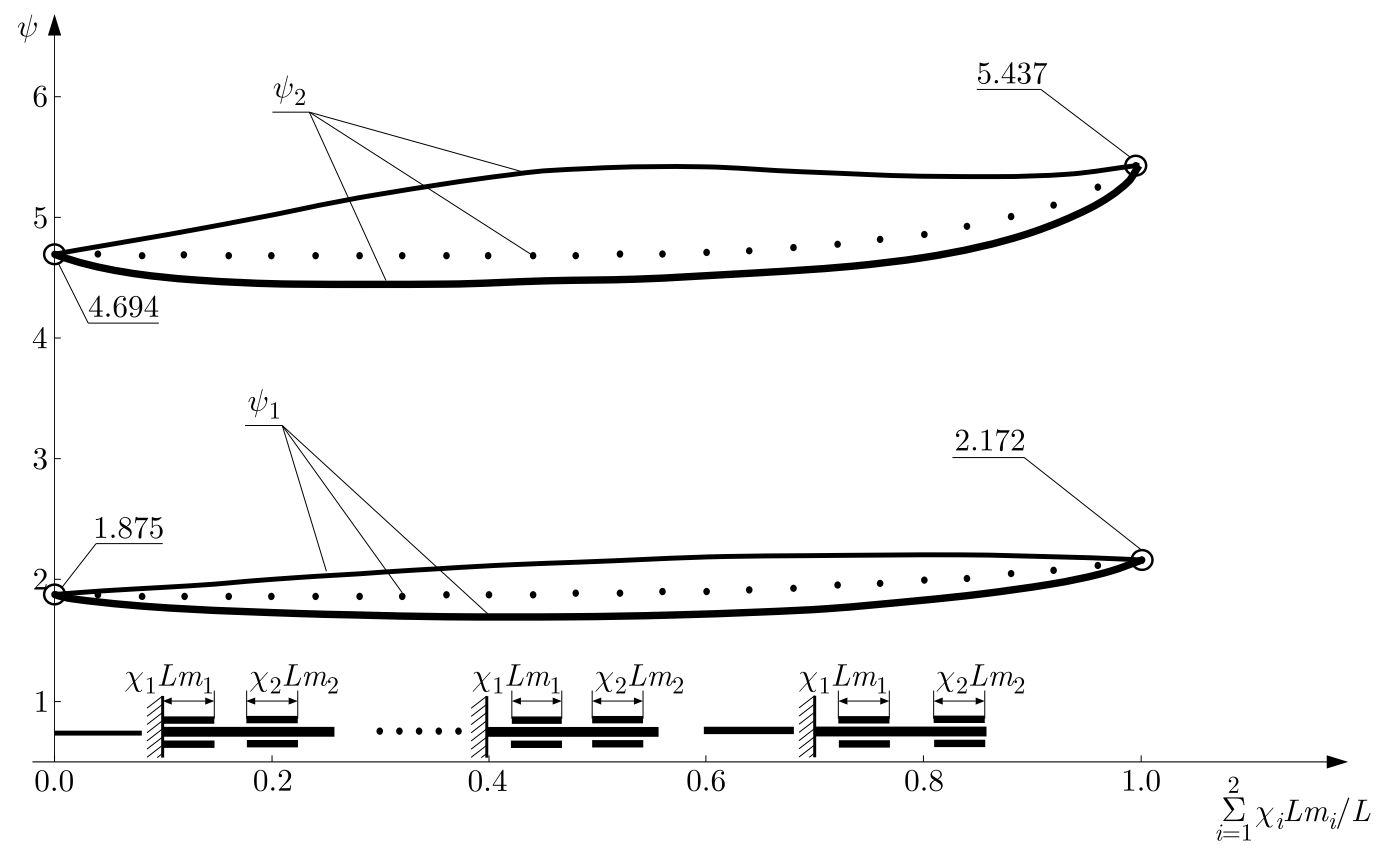

Fig. 10. The dimensionless frequencies $\psi$ for the converter with a double PTS segment, $\psi_{1}-$ first frequency, $\psi_{2}$ - second frequency 
Analyzing the obtained results, it can be stated that the highest values of the first dimensionless frequency, independent from PTS length, is acquired for the piezoelectric segment placed on the left end (Figs. 9 and 10). Similarly is with the second frequency for the converter with two segments. For the converter with one segment, the second frequency, depending on PTS length, takes the highest values for the converter with the segment placed either on the left end, or in the middle.

The dimensionless frequencies $\psi$ depend on the relative stiffness $\eta$ and the unit weight $\mu=\left(\rho_{b} A_{b}+2 \rho_{p} A_{p}\right) /\left(\rho_{b} A_{b}\right)$ of the beam and the PTS segment. In Figs. 11a and 11b, the influence of $\eta$ and $\mu$ on the $\psi$ value. Upon the received results, it can be stated that the dimensionless frequencies decrease with an increase in the parameters $\eta$ (Fig. 11a) and $\mu$ (Fig. 11b).

(a)

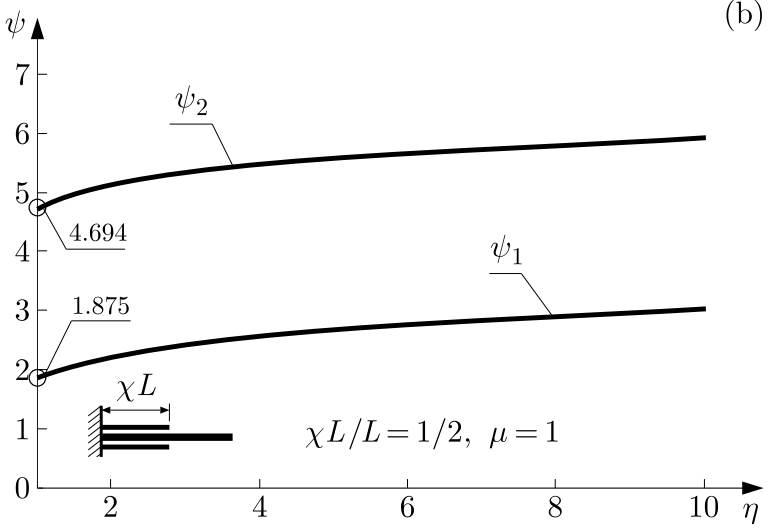

(b)

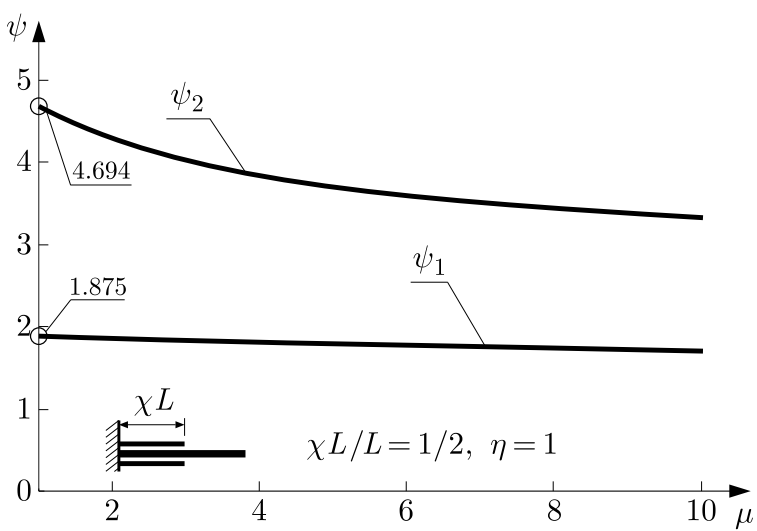

Fig. 11. The impact of relative stiffness (a) and relative unit (b) on the dimensionless frequency $\psi$ for the converter with a single PTS segment, $\psi_{1}$ - first frequency, $\psi_{2}$ - second frequency

The circular frequency $\omega[\mathrm{Hz}]$ of the transducer can be calculated from formula

$$
\omega=\frac{\psi^{2}}{2 \pi} \sqrt{\frac{E_{b} J_{b}}{\rho_{b} A_{b} L^{4}}}
$$

\section{Numerical calculations}

To confirm the correctness of the obtained analytical solutions (static deflection of the transducer and circular frequencies), it is necessary to perform numerical analyses. FEM simulations have been prepared and compared with the obtained analytical results. Numerical tests aimed at determining the bending line and circular frequencies of the actuators for arbitrarily assumed material constants and geometry. The tested converters, shown in Figs. 5-7, have been modelled using the FEM with the help of ANSYS (Mieszczak et al., 2006; Documentation for ANSYS, 2010). Plane components hve been described using a grid of quadrangular, eight-node finite elements, with increased concentration at critical points such as sharp corners, mounting points and places at which the mechanical load was applied. For the piezoelectric component, PLANE 223 type elements have been applied, and non-piezoelectric material has been meshed with PLANE 183 elements with steel material properties. In view of the fact that the actuators are usually made of piezoelectric ceramics, PZ26 has been used as a material of the piezoelectric component. The size of finite elements was $t_{p} / 4$. The plane issue has been solved for plane strain conditions.

In the calculations, the following geometrical and material data has been assumed: Young's modulus $E_{p}=7.7 \cdot 10^{10} \mathrm{~N} / \mathrm{m}^{2}, E_{b}=2.0 \cdot 10^{11} \mathrm{~N} / \mathrm{m}^{2}$; Poisson's ratio $\nu_{p}=0.3, \nu_{b}=0.33$; density $\rho_{p}=7700 \mathrm{~kg} / \mathrm{m}^{3}, \rho_{b}=7860 \mathrm{~kg} / \mathrm{m}^{3}$; piezoelectric strain coefficients $d_{31}=-1.28 \cdot 10^{-10} \mathrm{~m} / \mathrm{V}$, 
$d_{33}=3.28 \cdot 10^{-10} \mathrm{~m} / \mathrm{V}, d_{15}=3.27 \cdot 10^{-10} \mathrm{~m} / \mathrm{V}$; beam length $L=60 \mathrm{~mm}$; layers thickness $t_{p}=0.5 \mathrm{~mm}, t_{b}=1 \mathrm{~mm}$. The values of applied load are: electrode voltage $v=100 \mathrm{~V}$; force $F=100 \mathrm{~N}$; uniform external load $p=100 \mathrm{~N} / \mathrm{m}$. Coordinates $x_{i}, \chi_{i}$ (Figs. 5-8), defining the PTS and external loads application positions are given in Section 4.

\section{Results of tests}

In this part of the work, the deflection line of converters for which the resulting special solutions are given in Section 2.4 are graphically presented. The results obtained from the analytical solutions have been compared with FEM solutions. In the analytical equations, the material and geometrical data are identical as the data given in Section 3 was applied.

A comparison of analytical solution (2.18) with FEM for the cantilever converter of the single PTS segment (Fig. 5) is shown in Fig. 12.
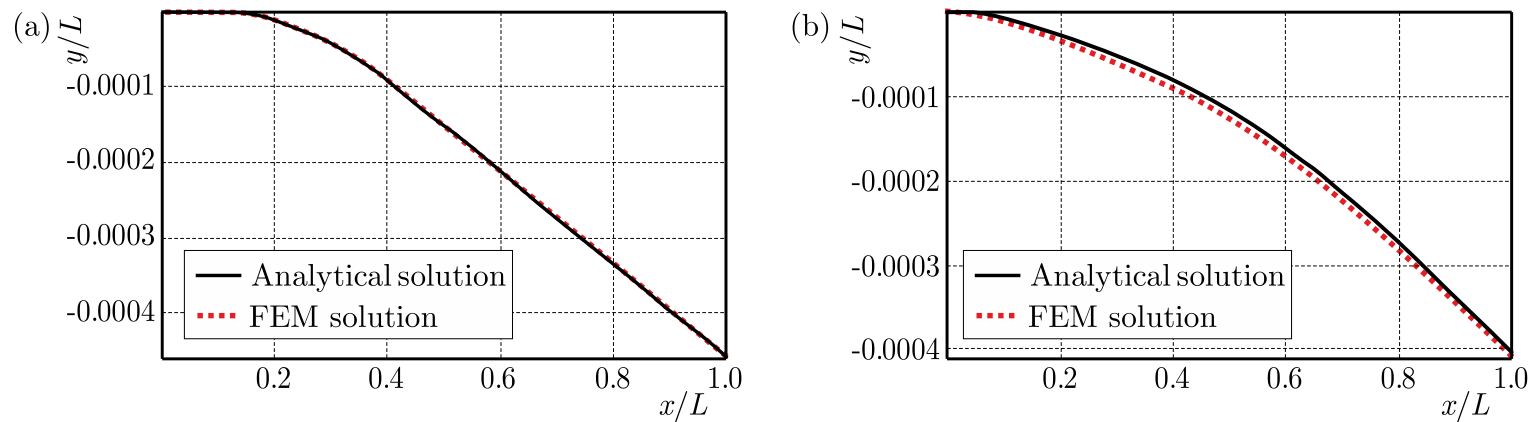

Fig. 12. Deflection of the cantilever converter with a single PTS segment for $x_{1}=1 / 12 L, x_{2}=5 / 12 L$ :

(a) subjected only to electrical voltage, $v=100 \mathrm{~V}, F=0$; (b) subjected only to force, $v=0 \mathrm{~V}$,

$$
F=100 \mathrm{~N}
$$

Figure 13 shows the deflection of the cantilever converter of double PTS segments (Fig. 6), for which the analytical solution is described by formula (2.22).

(a)

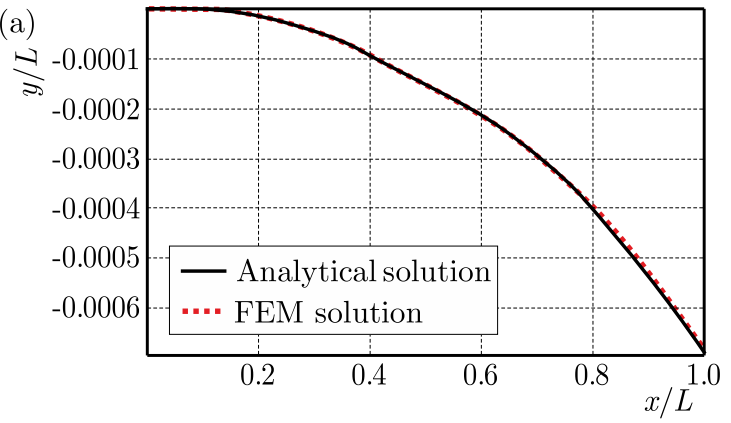

(b)

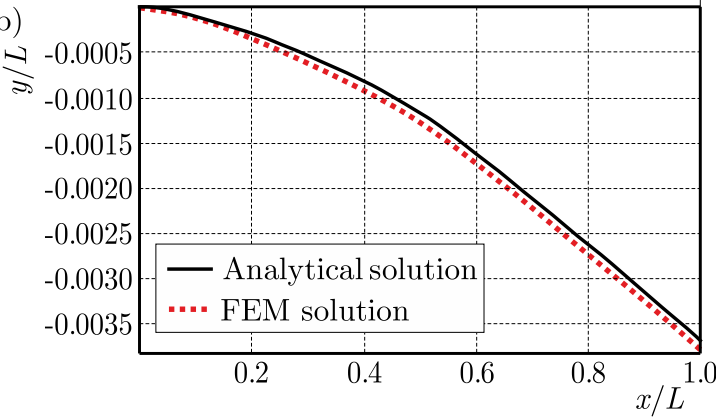

Fig. 13. Deflection of the cantilever converter with double PTS segments for $x_{1}=1 / 12 L, x_{2}=5 / 12 L$, $x_{3}=7 / 12 L, x_{4}=11 / 12 L$ : (a) subjected only to electrical voltage, $v_{1}=100 \mathrm{~V}, v_{2}=150 \mathrm{~V}, F=0$;

(b) subjected only to force, $v_{1}=v_{2}=0, F=100 \mathrm{~N}$

Figure 14 shows the strain of the cantilever converter subjected to a uniform external load $p$ of the single PTS segment (Fig. 7) for which the analytical solution is described by formula $(2.24)$.

Basing on the obtained results of static deflections of the converters, the qualitative and quantitative compliance of analytical and numerical solutions can be stated. Generally, the difference between the analytical and numerical solutions is approx. $1 \%$ for the electrical load, and $2-3 \%$ for the mechanical load. 

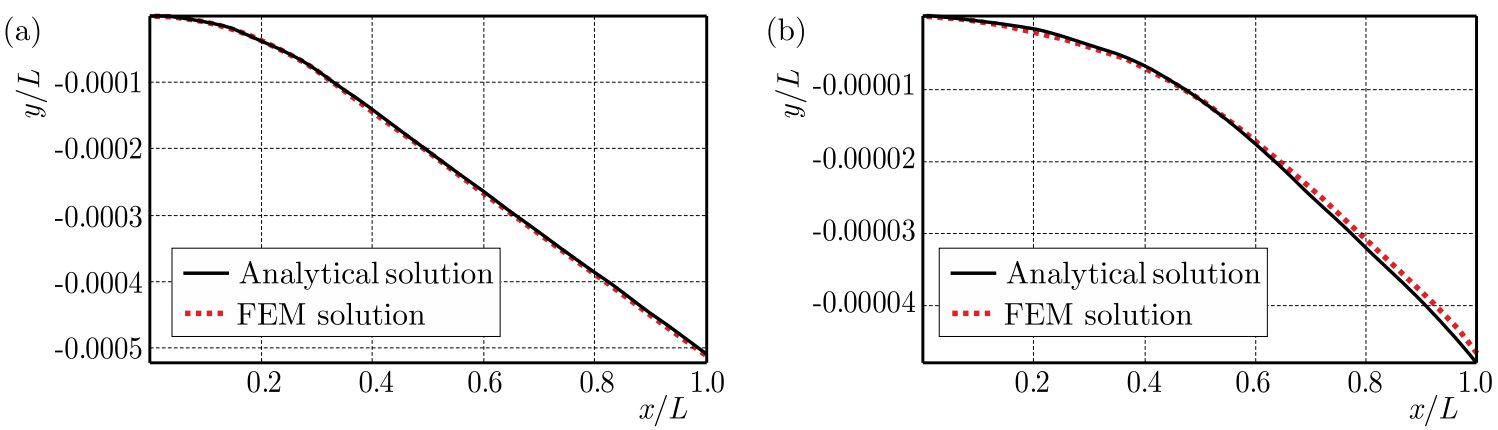

Fig. 14. Deflection of the cantilever converter subjected to a uniform external load $p$ for $x_{1}=1 / 3 L$, $x_{2}=2 / 3 L$ : (a) subjected only to electrical voltage, $v=-100 \mathrm{~V}, p=0$; (b) subjected only to uniform external load, $v=0 \mathrm{~V}, p=100 \mathrm{~N} / \mathrm{m}$

By means of FEM, it has been helpful to determine circular frequencies of the transducers with one (Fig. 7) and two (Fig. 6) PTS segments. The material and geometric data has been adopted identically as in the above-described static analysis. The obtained results (Table 1 ) have been compared to the analytical solution (formula (2.38) and Figs. 9 and 10).

Table 1. Comparison of the first two frequencies between the analytical and FEM results

\begin{tabular}{|c|c|c|c|c|c|c|}
\hline \multirow{3}{*}{$\begin{array}{c}\text { Mode } \\
\text { sequence }\end{array}$} & \multicolumn{3}{|c|}{$\chi L$} & \multicolumn{2}{|c|}{$\chi_{1} L$} & \\
\hline & & & $\chi=1 / 3$ & & & $\sum_{i=1}^{N} \chi_{i}=2 / 3$ \\
\hline & $\omega^{*}[\mathrm{~Hz}]$ & $\omega^{* *}[\mathrm{~Hz}]$ & Error [\%] & $\omega^{*}[\mathrm{~Hz}]$ & $\omega^{* *}[\mathrm{~Hz}]$ & Error [\%] \\
\hline 1 & 368.5 & $\overline{379.4}$ & 2.9 & 252.1 & 256.4 & 1.7 \\
\hline 2 & 1799.8 & 1837.7 & 2.1 & 1575.4 & 1543.8 & 2 \\
\hline
\end{tabular}

In the dynamical analysis as well as in the static analysis, a satisfactory compatibility of both obtained solutions has been indicated. The disparity in these results ranges less than $3 \%$.

\section{Summary and conclusions}

The paper deals with the issue of bending of three-layer piezoelectric actuators subjected to electric field and mechanical load. A general solution has been developed, based on the implementation of piezoelectric segments PTS to a homogeneous (one-layer) beam. The working mechanism and conditions for strain of the PTS segment have been determined. Basing on the general solution, for arbitrarily selected three different types of converters, special solutions have been developed (for the cantilever actuator of single and double PTS segments subjected to external force and the converter with a single PTS acted on by a uniform external load). Moreover, dynamical analysis of transducers has been performed. Also, a matrix whose determinant enables determination of the characteristic equation for the transducer with any amount of piezoelectric segments has been formulated. On the basis of characteristic equations, for the converter with one and two PTS, the natural frequencies and the influence of relative stiffness, size and placing of a segment on their value have been determined. The obtained analytical solutions have been compared with the developed FEM solution.

On the basis of the performed analytical and numerical tests, it is found that:

- the developed method involving the implementation of PTS segments into a homogeneous beam allows obtaining solutions for piezoelectric converters:

- of any either type of the external load, 
- of diverse lengths and heights of piezoelectric and non-piezoelectric layers,

- with any number of piezoelectric components;

- the obtained particular solutions allows determination of the deflection at any point of the converter;

- the maximum values of the first dimensionless frequency, independently of the length and number of PTS, are to be obtained for segments with mountings located closer;

- for a transducer with two PTS segments, the distribution of the second frequency is the same as for the first frequency;

- for a transducer with one piezoelectric segment, the second frequency depending on PTS length holds the highest value for either the converter with the segment located on the left end or for the one with the segment located in the middle;

- the dimensionless frequencies decrease with an increase in the relative stiffness and unit mass of the piezoelectric segment;

- the particular solutions of static behaviour confirm with the results obtained from FEM (for the electrical load the maximum difference is approx. 1\%, and for the mechanical load - approx. 3\%);

- a similar discrepancy between the analytical solution and FEM (less than 3\%) has been obtained while calculating circular frequencies.

\section{Appendix}

$$
\begin{aligned}
& \mathbf{M}_{1, i}^{12 \times 4}=\left[\begin{array}{cccc}
0 & 1 & 1 & 0 \\
\psi & 0 & 0 & \psi \\
\sin \left(\psi \kappa_{i}\right) & \cos \left(\psi \kappa_{i}\right) & \cosh \left(\psi \kappa_{i}\right) & \sinh \left(\psi \kappa_{i}\right) \\
\psi \cos \left(\psi \kappa_{i}\right) & -\psi \sin \left(\psi \kappa_{i}\right) & \psi \sinh \left(\psi \kappa_{i}\right) & \psi \cosh \left(\psi \kappa_{i}\right) \\
-\psi^{2} \sin \left(\psi \kappa_{i}\right) & -\psi^{2} \cos \left(\psi \kappa_{i}\right) & \psi^{2} \cosh \left(\psi \kappa_{i}\right) & \psi^{2} \sinh \left(\psi \kappa_{i}\right) \\
-\psi^{3} \cos \left(\psi \kappa_{i}\right) & \psi^{3} \sin \left(\psi \kappa_{i}\right) & \psi^{3} \sinh \left(\psi \kappa_{i}\right) & \psi^{3} \cosh \left(\psi \kappa_{i}\right) \\
0 & 0 & 0 & 0 \\
\vdots & \vdots & \vdots & \vdots \\
0 & 0 & 0 & 0
\end{array}\right] \\
& \mathbf{M}_{2, i}^{12 \times 4}=\left[\begin{array}{cccc}
0 & 0 & 0 & 0 \\
0 & 0 & 0 & 0 \\
0 & -1 & -1 & -\Lambda \psi \\
-\Lambda \psi & 0 & 0 & 0 \\
0 & \eta \Lambda^{2} \psi^{2} & -\eta \Lambda^{2} \psi^{2} & -\eta \Lambda^{3} \psi^{3} \\
\eta \Lambda^{3} \psi^{3} & 0 & 0 & -\sinh \left(\Lambda \psi \chi_{i}\right) \\
-\sin \left(\Lambda \psi \chi_{i}\right) & -\cos \left(\Lambda \psi \chi_{i}\right) & -\cosh \left(\Lambda \psi \chi_{i}\right) & -\Lambda \psi \cosh \left(\Lambda \psi \chi_{i}\right) \\
-\Lambda \psi \cos \left(\Lambda \psi \chi_{i}\right) & \Lambda \psi \sin \left(\Lambda \psi \chi_{i}\right) & -\Lambda \psi \sinh \left(\Lambda \psi \chi_{i}\right) & -\eta \Lambda^{2} \psi^{2} \sinh \left(\Lambda \psi \chi_{i}\right) \\
\eta \Lambda^{2} \psi^{2} \sin \left(\Lambda \psi \chi_{i}\right) & \eta \Lambda^{2} \psi^{2} \cos \left(\Lambda \psi \chi_{i}\right) & -\eta \Lambda^{2} \psi^{2} \cosh \left(\Lambda \psi \chi_{i}\right) & 0 \\
\eta \Lambda^{3} \psi^{3} \cos \left(\Lambda \psi \chi_{i}\right) & -\eta \Lambda^{3} \psi^{3} \sin \left(\Lambda \psi \chi_{i}\right) & -\eta \Lambda^{3} \psi^{3} \sinh \left(\Lambda \psi \chi_{i}\right) & -\eta \Lambda^{3} \psi^{3} \cosh \left(\Lambda \psi \chi_{i}\right) \\
0 & 0 & 0 & 0 \\
0 & 0 & 0 & 0
\end{array}\right]
\end{aligned}
$$




$$
\begin{aligned}
& \mathbf{M}_{3, i}^{12 \times 4}=\left[\begin{array}{cccc}
0 & 0 & 0 & 0 \\
\vdots & \vdots & \vdots & \vdots \\
0 & 1 & 1 & 0 \\
\psi & 0 & 0 & \psi \\
0 & -\psi^{2} & \psi^{2} & 0 \\
-\psi^{3} & 0 & 0 & \psi^{3} \\
-\psi^{2} \sin \left(\psi \phi_{i}\right) & -\psi^{2} \cos \left(\psi \phi_{i}\right) & \psi^{2} \cosh \left(\psi \phi_{i}\right) & \psi^{2} \sinh \left(\psi \phi_{i}\right) \\
-\psi^{3} \cos \left(\psi \phi_{i}\right) & \psi^{3} \sin \left(\psi \phi_{i}\right) & \psi^{3} \sinh \left(\psi \phi_{i}\right) & \psi^{3} \cosh \left(\psi \phi_{i}\right)
\end{array}\right] \\
& \mathbf{B}^{12 \times 12}=\left[\begin{array}{cccccccc}
0 & 0 & 0 & 0 & 0 & \cdots & \cdots & 0 \\
\vdots & \vdots & \vdots & \vdots & \vdots & \cdots & \cdots & \vdots \\
0 & \psi^{2} & -\psi^{2} & 0 & 0 & . & . & 0 \\
\psi^{3} & 0 & 0 & -\psi^{3} & 0 & . & . & 0
\end{array}\right] \\
& \mathbf{C}_{i}^{12 \times 12}=\left[\begin{array}{cccccc}
0 & \cdots & -\sin \left(\psi \phi_{i}\right) & -\cos \left(\psi \phi_{i}\right) & -\cosh \left(\psi \phi_{i}\right) & -\sinh \left(\psi \phi_{i}\right) \\
0 & \cdots & -\psi \cos \left(\psi \phi_{i}\right) & \psi \sin \left(\psi \phi_{i}\right) & -\psi \sinh \left(\psi \phi_{i}\right) & -\psi \cosh \left(\psi \phi_{i}\right) \\
\vdots & \ddots & \vdots & \vdots & \vdots & \vdots \\
0 & \cdots & 0 & 0 & 0 & 0
\end{array}\right]
\end{aligned}
$$

\section{References}

1. Askari Farsangi M.A., Saidi A.R., Batra R.C., 2013, Analytical solution for free vibrations of moderately thick hybrid piezoelectric laminated plates, Journal of Sound and Vibration, 332, 5981-5998

2. Berlincourt D.A., Curran D.R., Jaffe H., Mason W.P., 1964, Physical Acoustics, Principles and Methods, Academic Press, New York

3. Bleustein J.L., Tiersten H.F., 1968, Forced thickness-shear vibrations of discontinuously plated piezoelectric plates, Journal of the Acoustical Society of America, 43, 6, 1311

4. Buchacz A., PŁaczek M., 2009, Damping of mechanical vibrations using piezoelements, including influence of connection layer's properties, on the dynamic characteristic, Solid State Phenomena, 147/149, 869-875

5. Busch-Vishniac I.J., 1999, Electromechanical Sensors and Actuators, Springer-Verlag

6. Chen W.Q., Xu R.Q., Ding H.J., 1998, On free vibration of a piezoelectric composite rectangular plate, Journal of Sound and Vibration, 218, 4, 741-748

7. Clare R.L., Fuller Ch.R., Wicks A., 1991, Characterization of multiple piezoelectric actuators for structural excitation, Journal of Acoustical Society of America, 90, 346-357

8. Curie P.J., Curie J., 1880, Crystal physics-development by pressure 0/ polar electricity in hemihedral crystals with inclined faces (in French), Comptes rendus hebdomadaires des sances de l'Acadmie des sciences, 294, pp. 91

9. Djojodihardjo H., Jafari M., Wiriadidjaja S., Ahmad K.A., 2015, Active vibration suppression of an elastic piezoelectric sensor and actuator fitted cantilevered beam configurations as a generic smart composite structure, Composite Structures, 132, 848-863

10. Documentation for ANSYS, 2010, Coupled-Field Analysis Guide

11. Fertis D.G., 1996, Advanced Mechanics of Structures, Marcel Dekker, New York

12. KaWAi H., 1969, The piezoelectricity of poly (vinylidene fluoride), Japanese Journal of Applied Physics, 8, 975

13. Liu X., Wang X., Zhao H., Du Y., 2014, Myocardial cell pattern on piezoelectric nanofiber mats for energy harvesting, Journal of Physics: Conference Series, 557, 012057 
14. Mahmoud A.A., Nassar M.A., Free vibration of a stepped beam with two uniform and/or tapered parts, Mechanics and Mechanical Engineering, 4, 2, 165-181

15. Mieczkowski G., 2016, Electromechanical characteristics of piezoelectric converters with freely defined boundary conditions and geometry, Mechanika, 22, 4, 265-272

16. Mieszczak Z., Krawczuk M., Ostachowicz W., 2006, Interaction of point defects in piezoelectric materials - numerical simulation in the context of electric fatigue, Journal of Theoretical and Applied Mechanics, 44, 4, 650-665

17. PARK J.K., Moon W.K., 2005, Constitutive relations for piezoelectric benders under various boundary conditions, Sensors and Actuators A, 117, 159-167

18. Pietrzakowski M., 2000, Multiple piezoceramic segments in structural vibration control, Journal of Theoretical and Applied Mechanics, 38, 378-393

19. Pietrzakowski M., 2001, Active damping of beams by piezoelectric system: effects of bonding layer properties, International Journal of Solids and Structures, 38, 7885-7897

20. PrzybyŁowicz P.M., 1999, Application of piezoelectric elements to semi-adaptive dynamic eleminator of torsional vibration, Journal of Theoretical and Applied Mechanics, 37, 2, 319-334

21. Raeisifard H., Bahrami M.N., Yousefi-Koma A., Fard H.R., 2014, Static characterization and pull-in voltage of a micro-switch under both electrostatic and piezoelectric excitations, European Journal of Mechanics A/Solids, 44, 116-124

22. Rahmoune M., Osmont D., 2010, Classic finite elements for simulation of piezoelectric smart structures, Mechanika, 86, 6, 50-57

23. Rajabi A.H., Jaffe M., Arinzeh T.J., 2015, Piezoelectric materials for tissue regeneration: A review, Review Article, Acta Biomaterialia, 24, 15, 12-23

24. Rouzegar J., Abad F., 2015, Free vibration analysis of FG plate with piezoelectric layers using four-variable refined plate theory, Thin-Walled Structures, 89, 76-83

25. Smits J.G., Dalke S.I., Cooney T.K., 1991, The constituent equations of piezoelectric bimorphs, Sensors and Actuators A, 28, 41-61

26. Ştefănescu D.M. 2011, Piezoelectric Force Transducers (PZFTs), [In:] Handbook of Force Transducers, 109-130

27. Tylikowski A., 1993, Stabilization of beam parametric vibrations, Jurnal of Theoretical and Applied Mechanics, 31, 3, 657-670

28. Tzou H.S., 1999, Piezoelectric Shells: Distributed Sensing and Control of Continua, Kluwer Academic Publishers, Dordrecht

29. WAng Q., Cross L.E., 1999, Constitutive equations of symmetrical triple-layer piezoelectric benders, IEEE Transactions on Ultrasonics, Ferroelectronics, and Frequency Control, 46, $1343-1351$

30. Xiang H.J., Shi Z.F., 2008, Static analysis for multi-layered piezoelectric cantilevers, International Journal of Solids and Structures, 45, 1, 113-128

Manuscript received October 6, 2015; accepted for print May 6, 2016 